\title{
Pharmaciana
}

Vol.9, No.2, Nov 2019, Page. 299-306

ISSN: 2088 4559; e-ISSN: 24770256

DOI: $10.12928 /$ pharmaciana.v9i2.13070

\section{Entrapment efficiency and drug loading of curcumin nanostructured lipid carrier (NLC) formula}

\author{
Rabima Rabima*, Metri Purnama Sari \\ Faculty of Pharmacy, University of 17 Agustus 1945, Jakarta \\ Submitted: 08-05-2019 \\ Reviewed: 09-05-2019 \\ Accepted: 25-11-2019
}

\begin{abstract}
Curcumin is a chemical compound that has low solubility and bioavailability and, for these reasons, limited biological effectiveness. For improvement, the solubility needs to be increased by nanotechnology and nanoparticles, among others. Nanostructured Lipid Carrier (NLC) is a new drug delivery system that offers several advantages, including a significant increase in drug solubility and entrapment efficiency. This study aimed to formulate curcumin into curcumin-loaded NLC preparation and determine its characteristics, absorption efficiency, and drug loading. The formulation used evaporation and solvent diffusion methods with three different concentrations, namely 5\%,10\%, and $15 \%$. Spectrophotometry and HPLC were employed to test the absorption efficiency and drug loading capacity. The results showed that the curcumin-loaded NLC preparation containing $10 \%$ curcumin had stable characteristics and produced particles sized $17.4 \mathrm{~nm}$ with a polydispersity index of 0.574 and zeta potential of $-63.43 \mathrm{mV}$. Based on the spectrophotometry results, the entrapment efficiency was $93.212 \%$, and the drug loading capacity was $0.708 \%$. Meanwhile, the HPLC showed that the entrapment efficiency was $93.007 \%$, and the drug loading capacity was $0.795 \%$.
\end{abstract}

Keywords: drug loading, absorption efficiency, curcumin, NLC

\section{*Corresponding author:}

Rabima

Faculty of Pharmacy, University of 17 Agustus 1945, Jakarta

Email: Rabima86@gmail.com 


\section{INTRODUCTION}

Curcumin is a chemical compound responsible for the yellow color in turmeric and has a broad therapeutic effect (Soni et al., 2011). It has several unfavorable properties such as low solubility and low bioavailability (Wang et al., 2009; Yang et al., 2007), and this combination negatively affects its biological effectiveness (Shaikh et al., 2009). Therefore, developing formulas to increase the bioavailability of this natural compound becomes necessary (Ajazudin, 2010).

Nanotechnology and nanoparticles are two approaches known to enhance the poor biopharmaceutical properties of curcumin by increasing its aqueous solubility (Torchilin, 2009; Ruenraroengsak et al., 2010; Sultana et al., 2013). Nanostructured Lipid Carrier (NLC) is a drug delivery system with several benefits, which include increased solubility of less dissolvable drugs, physical stability, and efficiency entrapment, as well as reduced skin irritation and ease of manufacture and improvement (Sanad et al., 2010; Kaur et al., 2015; Jain and Jain, 2010).

Based on the description above, research on the entrapment efficiency and drug loading of the curcumin-loaded NLC formula becomes necessary. This study is expected to obtain a stable preparation, with improved \%entrapment efficiency and drug loading.

\section{MATERIALS AND METHODS \\ Materials}

The tools used in this study were batch heater, hotplate, magnetic stirrer, homogenizer (IKA Ultra-Turrax), 130-Watt ultrasonic processor $20 \mathrm{kHz}$ (Cole-Parmer), particle size analyzer (Delsa Nano Beckman Coulter), UV-1700 UV/Vis spectrophotometer (Shimadzu), Fourier-Transform Infrared (FTIR) Spectrophotometer (Shimadzu), JEOL JEM 1400 transmission electron microscope for the High Performance Liquid Chromatography (Shimadzu LC-20AT(HPLC)), and glassware. The materials included curcumin (Merck), cholesterol (Merck), oleic acid (Sigma-Aldrich), Tween 80 (Merck), distilled water, potassium bromide (Merck), ethanol (Merck), and acetone (Merck).

\section{Curcumin-loaded NLC formulation}

Curcumin-loaded NLC was created by evaporation and solvent diffusion in aqueous systems. The stages were as follows: $60 \mathrm{mg}$ of a mixture of cholesterol and $15 \%$ oleic acid (AO) was dissolved in a mixture of acetone $(3 \mathrm{~mL})$ and ethanol $(3 \mathrm{~mL})$, then heated at $60^{\circ} \mathrm{C}$. Curcumin was added to the lipid phase (drug/lipid 10\%), and the heating temperature was maintained at $60^{\circ} \mathrm{C}$. Meanwhile, the aqueous phase was prepared by mixing distilled water $(60 \mathrm{~mL})$ with tween 80 (concentration of $1 \%$ ) and heating this mixture at a temperature of $60^{\circ} \mathrm{C}$. Immediately, the aqueous mixture was added to the lipid mixture, and then homogenized using the IKA Ultra-Turrax homogenizer at $800 \mathrm{rpm}$ for 2 to 4 minutes. Then, the pre-emulsion mixture was screened using ultrasound for 20 minutes at an amplitude of 20\%. Finally, the NLC disperse was cooled to room temperature $\left(25^{\circ} \mathrm{C}\right)$ and stored at $4^{0} \mathrm{C}$ (Emami et al., 2012).

\section{Characterization of curcumin-loaded NLC}

Curcumin-loaded NLC was characterized by particle size, zeta potential, and polydispersity index using Delsa ${ }^{\mathrm{TM}}$ Nano C (Beckman Coulter, Inc.). The structure and morphology of the final product were observed under the JEOL JEM 1400 transmission electron microscope at an accelerating voltage of $100 \mathrm{kV}$.

\section{Identification of curcumin-loaded NLC}

The curcumin compounds in curcumin-loaded NLC was identified by Fourier-Transform Infrared (FT-IR) Spectroscopy. Afterward, the FTIR spectrum of the liquid samples was analyzed by dripping the sample onto $\mathrm{KBr}$ powder, followed by drying and homogeneous grinding. The IR spectrum was checked at the wavenumbers of 400 to $4000 \mathrm{~cm}^{-1}$. 
Absorption efficiency analysis

The efficiency of curcumin-loaded NLC was determined by centrifugation at $12,000 \mathrm{rpm}$ for 60 minutes. The supernatant was decanted and then measured using a UV-Vis spectrophotometer at a wavelength of $423 \mathrm{~nm}$ and by High-Performance Liquid Chromatography (HPLC). As for the drug loading, it was analyzed from the curcumin content in the NLC using a spectrophotometer at a wavelength of $423 \mathrm{~nm}$ and by High-Performance Liquid Chromatography (HPLC), with acetonitrile and $2 \%$ acetic acid as the mobile phase. The absorption efficiency (\% EE) and drug loading (\% DL) were calculated using the following equations:

$\% \mathrm{EE}=\left(\frac{\mathrm{A}-\mathrm{B}}{\mathrm{A}}\right) \times 100 \%$.
$\% \mathrm{DL}=\left(\frac{\mathrm{C}}{A+D}\right) \times 100 \%$.

$\mathrm{A}=$ The amount of curcumin added

$\mathrm{B}=$ The amount of curcumin in the supernatant

$\mathrm{C}=$ The amount of curcumin added (DL)

$\mathrm{D}=$ The number of excipients added

\section{RESULTS AND DISCUSSION}

The curcumin-loaded NLC was formulated by evaporation and solvent diffusion with several variations in curcumin's concentration (active substance), i.e., $5 \%$ or $3 \mathrm{mg}$ curcumin (Formula 1), $10 \%$ or $6 \mathrm{mg}$ (Formula 2), and $15 \%$ or $9 \mathrm{mg}$ (Formula 3 ).

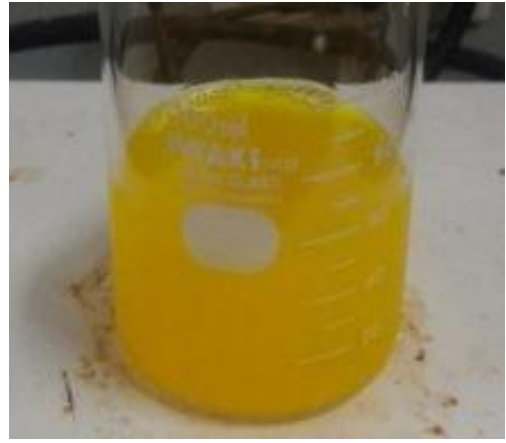

Figure1. The curcumin-loaded NLC formulated with $10 \%$ curcumin

Cholesterol was used as a solid lipid because it can increase the drug-carrying system to specifically target cancer cells (Emami et al., 2012), while oleic acid was chosen as a liquid lipid because it can produce NLC with a higher entrapment efficiency than any drugs that do not contain oleic acid. Tween 80 was selected as an emulsifier because, if enriched with this surfactant, the resultant NLC has a high entrapment efficiency (Attama and Müller-Goymann 2008). The combination of homogenization and ultrasonication techniques has several advantages, including nanoparticle preparations with smaller particle size and the use of simple and effective equipment for a scale of a laboratory (Asawale et al., 2014). Formula 2 (10\%) produced curcumin-loaded NLC with clear yellow color, no sediment, no foaming, and favorable homogeneity (Figure 1). Meanwhile, Formula 1 and 2, each using 5\% and 15\% curcumin, yielded yellow liquid containing sediment. The presence of this deposit in the final product shows that the small particles are not well dispersed; in other terms, the curcumin-loaded NLC prepared using Formula 1 (5\%) and Formula 3 (15\%) cannot be used for further testing. 


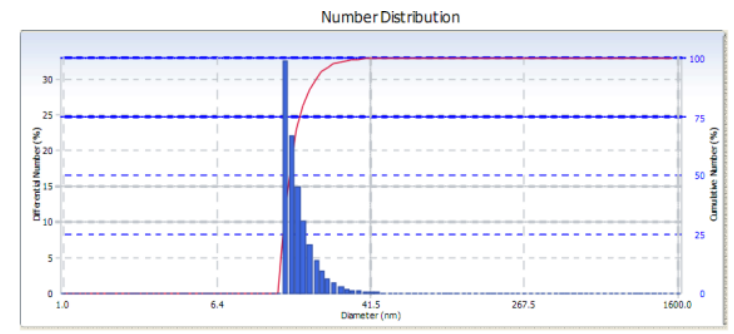

Figure 2. The PSA analysis results of the produced curcumin-loaded NLC

Particle size is an essential characteristic of nanoparticles because it determines the stability of the nanoparticle system. Smaller particle size increases the surface area and, consequently, leads to high solubility, making the particles easier to be absorbed in the body (Awad et al., 2008). The diameter size of the produced curcumin-loaded NLC was $17.4 \mathrm{~nm}$. It proves that the oleic acid content in NLC initiates nano lipid formation with smaller particle sizes. Similar results are reported in other studies (Hu et al. 2008; Agrawal et al., 2010; Tiwari and Pathak, 2011).

The polydispersity index represents the distribution of nanoparticle in a dosage form. In this study, the polydispersity index started from 0.01 to $0.5-0.7$ for monodispersed particles. Based on the results of the particle size analyzer (Figure 2), the polydispersity index of the curcumin-loaded NLC was 0.574 . Monodisperse nanoparticles can improve the stability of the nanoparticle system because they show the size, shape, and weight of homogeneous particles. A higher polydispersity index means that more particles are aggregated, or in other words, the preparation is increasingly unstable.

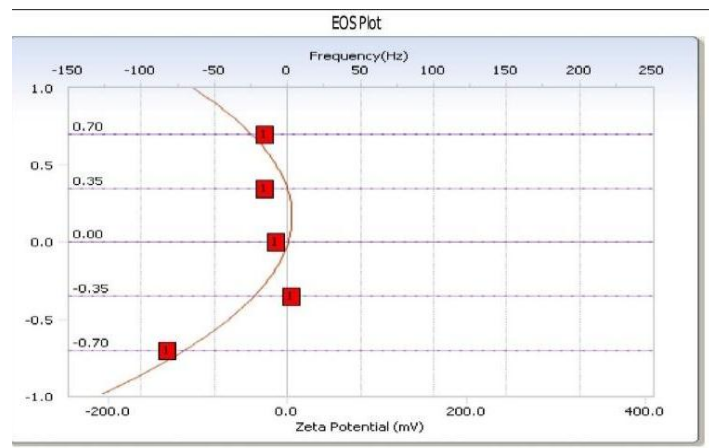

Figure 3. The zeta potential analysis of the curcumin-loaded NLC

The results showed that the zeta potential of the prepared curcumin-loaded NLC was -63.43 $(\mathrm{mV})$. Zeta potential represents the aggregation of the preparation, i.e., a high zeta potential, both negative and positive, indicates a colloidal system that tends to be stable and can prevent particles from aggregating. In general, particles with a zeta potential higher (more positive) than $+30 \mathrm{mV}$ or lower (more negative) than $-30 \mathrm{mV}$ are considered stable (Rosli et al., 2015). 


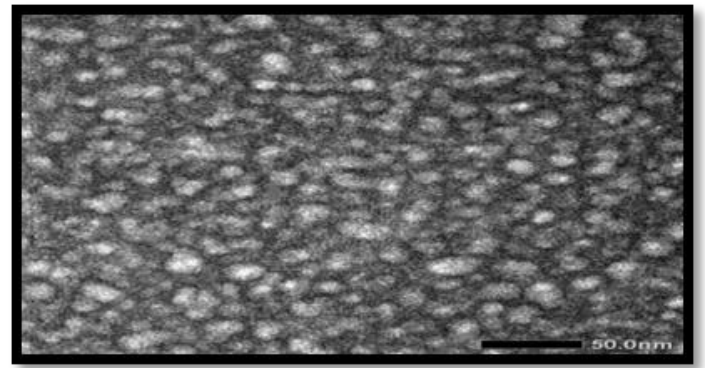

Figure 4. The morphological analysis results of curcumin-loaded NLC, as observed under a transmission electron microscope

The morphology of the NLC particle was examined under a transmission electron microscope. Based on the observation results, the curcumin-loaded NLC particles were round with a smooth surface and no appearance of particle aggregation (Figure 4). This finding is consistent with Almoussalam (2015), which suggests that nanoparticles with lipids as carriers have a round structure with a smooth surface, regardless of the type of the lipid used. The size of the curcumin nanoparticles obtained was $68.18 \mathrm{~nm}$, which is similar to the size of curcuminoid nanoparticles prepared in Mujib (2011), that is, $70.21 \mathrm{~nm}$. These results prove that the oleic acid content in NLC forms nano lipids with smaller particle sizes.

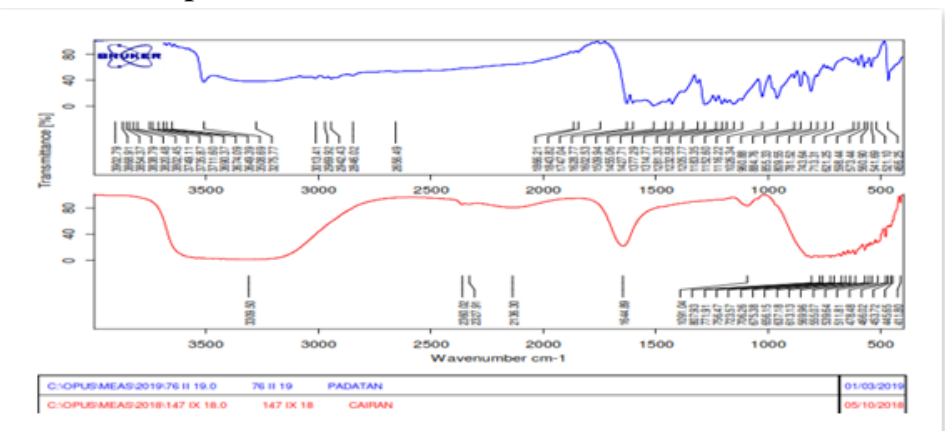

Figure 5. The identification results of compounds in curcumin-loaded NLC by FTIR (the blue curve is the NLC spectrum, and the red one is curcumin)

The infrared spectra of pure curcumin and curcumin-loaded NLC were compared (Figure 5). Based on the observation results, the standard infrared spectrum for curcumin was as follows: 1628 $\mathrm{cm}^{-1}$ (for $\mathrm{C}=\mathrm{C}$ aromatic), $1427 \mathrm{~cm}^{-1}$ (for CC aromatic), $1602 \mathrm{~cm}^{-1}$ (for conjugated diene), $1509 \mathrm{~cm}^{-1}$ (for $\mathrm{C}=\mathrm{C}$ ketones), 3200-3508 $\mathrm{cm}^{-1}$ (for $\mathrm{OH}$ bonded to the aromatic group), $809 \mathrm{~cm}^{-1}$ and $855 \mathrm{~cm}^{-1}$ (for substituted aromatic ring). Meanwhile, the infrared spectrum of the NLC sample did not have the main peaks of curcumin $\left(1628,1427,1602,1509,3200-3508,809-855 \mathrm{~cm}^{-1}\right)$, but those of oleic acid were apparent, as marked by the wavenumbers of $1644 \mathrm{~cm}^{-1}$ (for $\mathrm{C}=\mathrm{C}$ alkene) and $3309 \mathrm{~cm}^{-1}$ (OH-carboxylic). The different spectra between the standard curcumin and curcumin-loaded NLC samples prove that curcumin has been encapsulated by NLC. 
Table I. The entrapment efficiency and drug loading of curcumin-loaded NLC, as measured using a UV-VIS Spectrophotometer

\begin{tabular}{cc}
\hline$\%$ Entrapment Efficiency & $\%$ Drug Loading \\
\hline $93.212 \%$ & $0.708 \%$ \\
\hline
\end{tabular}

Entrapment efficiency determines how much percentage of the active substance is absorbed in the NLC compared to the total curcumin added. The absorbance values showed that the concentration of curcumin was absorbed, while the absorption efficiency of the curcumin-loaded NLC was identified using a linear equation of the standard curcumin curve. The absorption efficiency was 93.212\% (Table I). Such a high absorption efficiency is likely to increase drug release (Sonaje et al., 2007). The drug loading was measured by dividing the amount of curcumin absorbed in the nanoparticle system by the total amount of solution. This calculation yielded a drug loading of $0.708 \%$. A nanoparticulate system ideally has a high loading capacity that can reduce the quantity of material used for drug delivery.

Table II. The absorption efficiency and drug loading of curcumin-loaded NLC-, as measured by High-Performance Liquid Chromatography (HPLC)

\begin{tabular}{cc}
\hline \% Entrapment Efficiency & \% Drug Loading \\
\hline $93.007 \%$ & $0.795 \%$ \\
\hline
\end{tabular}

A drug delivery system must have a high loading capacity. Entrapment efficiency is generally expressed in percent of drug absorption. In this study, the entrapment efficiency was determined by centrifuging curcumin-loaded NLC samples and measuring the supernatant by HPLC. The results showed that the entrapment efficiency of curcumin-loaded NLC was $93.07 \%$. This figure is close to $100 \%$, indicating that curcumin was entirely encapsulated by NLC. Severino et al. (2015) formulate NLC with $1 \%$ surfactant and produce $90 \%$ entrapment efficiency. The amount of curcumin absorbed in the nanoparticle system was divided by the total amount of solution to quantify loading capacity. This calculation result showed that the drug loading of the produced curcumin-loaded NLC was $0.795 \%$. An ideal nanoparticle system has a high loading capacity, and in this condition, the quantity of materials used for drug delivery can be reduced.

\section{CONCLUSION}

Curcumin-loaded NLC that is prepared with $10 \%$ curcumin has stable characteristics, a particle size of $17.4 \mathrm{~nm}$, a polydispersity index of 0.574 , and zeta potential of $-63.43 \mathrm{mV}$. Morphologically, it is round in shape with a smooth surface. Based on the spectrophotometry results, this preparation has an entrapment efficiency of $93.212 \%$ and a drug loading capacity of $0.708 \%$. Meanwhile, the HPLC shows that the entrapment efficiency is $93.007 \%$, and the drug loading capacity is $0.795 \%$.

\section{ACKNOWLEDGMENT}

The authors would like to thank the Indonesian Herbal Nanotech and PSSP Biopharmaca Laboratory, Bogor LPPM-LPPM, for providing laboratory facilities and infrastructure required in this study.

\section{REFERENCES}

Agrawal, K.C., Petkar, K.K., Sawant., 2010, Development, evaluation and clinical studies of Acitretin loaded nanostructured lipid carriers for topical treatment of psoriasis, International Journal of Pharmaceutics, 401: 93-102. 
Almoussalam., 2015, Characterization, purification and stability of gold nanoparticles, International Journal of Pharmaceutical Sciences, 7 (2): 211-220.

Ajazudin., S.S., 2010, Applications of novel drug delivery system for herbal formulations, Fitoterapia, 81(7):680-9.

Asawale, R.H., Meshram, J.H., Kumbhar, V.B., 2014, Solid lipid nanoparticles as drug delivery system: an overview, Pharmacie Globale: International Journal of Comprehensive Pharmacy: 5(1):1-10.

Attama, A.A., and Müller-Goymann, C.C., 2008, Effect of Beeswax Modification on the Lipid Matrix and Solid Lipid Nanoparticle Crystallinity, Colloids and Surfaces A: Physicochemical and Engineering Aspects, 315 (1-3), Elsevier: 189-95.

Awad, T., Helgason, T., Kristbergsson, K., Decker, E.A., Weiss, J., McClements, D.J., 2008, Solid lipid nanoparticles as delivery systems for bio-active food components, Food Biophysics, 3:146-154.

Emami, J., Rezazadeh, M., Varshosaz, J., Tabbakhian, M., Aslani, A., 2012, Formulation of LDL targeted nanostructured lipid carriers loaded with paclitaxel: A detailed study of preparation, freeze drying condition and invitro cytotoxicity. Journal of Nanomaterials, 1:1-10.

Fang, C, L, A., Al-Suwayeh, S., Fang, J.Y., 2013, Nanostructured Lipid Carriers (NLCs) for Drug Delivery and Targeting, Recent Patents on Nanotechnology, 7(1): 41-55.

Hu, F.Q., Zhang, Y., Du, Y.Z., Yuan, H., 2008, Nimodipine loaded lipid nanospheres prepared by solvent diffusion method in a drug saturated aqueous system, International Journal of Pharmaceutics, 348(1-2):146-52.

Jain, S.K., and Jain, N.K., 2010, Multiparticulate carriers for sun-screening agents, International Journal of Cosmetic Science, 32(2): 89-98.

Kaur, S., Nautyal, U., Singh, R., Singh, S., Devi, A., 2015, Nanostructure Lipid Carrier (NLC): the new generation of lipid nanoparticles, Asian Pacific Journal of Health Sciences, 2(2): 76-93.

Mujib, M.A., 2011, Pencirian nanopartikel kurkuminoid tersalut asam lemak padat, Master Thesis, Institut Pertanian Bogor, Bogor, Indonesia.

Rosli, N.A., Hasham, R., Aziz, A., Aziz, R., 2015, Formulation and characterization of nanostructured lipid carrier encapsulated Zingiber zerumbet oil using ultrasonication technique, Journal of Advanced Research in Applied Mechanics,11(1): 16-23.

Ruenraroengsak, P., Cook, J.M., Florence, A.T., 2010, Nanosystem drug targeting: facing up to complex realities, Journal of controlled Release, 141(3): 265-276.

Sanad, R.A., Abdelmalak, N.S., Elbayoomy, T.S., Badawi, A.A., 2010, Formulation of a novel oxybenzone-loaded nanostructured lipid carriers (NLCs), AAPS Pharm SciTech, 11(4): 16841694.

Severino, P., Andreani, T., Chaud, M.V., Benites, C.I., Pinho, S.C., Souto, E.B., 2015, Essential oils as active ingredients of lipid nanocarriers for chemotherapeutic use, Current Pharmaceutical Biotechnology, 16(4): 365-370. 
Shaikh, J., Ankola, D.D., Beniwal, V., Singh, D., Kumar, M.N., 2009, Nanoparticle encapsulation improves oral bioavailability of curcumin by at least 9-fold when compared to curcumin administered with piperine as absorption enhancer, European Journal of Pharmaceutical Sciences, 37(3-4): 223-230.

Sonaje, K., Italia, J.L., Sharma, G., Bhardwaj, V., Tikoo, K., Kumar, M.N., 2007, Development of biodegradable nanoparticles for oral delivery of ellagic acid and evaluation of their antioxidant efficacy against cyclosporine A-induced nephrotoxicity in rats. Pharmaceuticals Research, 24(5): 899-908.

Soni, H., Patel, S.S., Govind, N., Singhai, A.K., 2011, Qualitative and quantitative profile of curcumin from ethanolic extract of Curcuma longa, International Research Journal of Pharmacy, 2(4): $180-184$.

Sultana, S., Khan, M.R., Kumar, M., Kumar, S., Ali, M., 2013, Nanoparticles-mediated drug delivery approaches for cancer targeting: a review, Journal of Drug Targeting, 21(2): 107-125.

Tiwari, R., and Pathak, K., 2011, Nanostructured lipid carrier versus solid lipid nanoparticles of simvastatin: comparative analysis of characteristics, pharmacokinetics and tissue uptake, International Journal of Pharmaceutics, 415(1-2): 232-243.

Torchilin, V., 2009, Multifunctional and stimuli-sensitive pharmaceutical nanocarriers, European Journal of Pharmaceutics and Biopharmaceutics, 71(3), 431-444.

Wang, Y., Lu, Z., Lv, F., Bie, X., 2009, Study on microencapsulation of curcumin pigments by spray drying, European Food Research and Technology, 229(3): 391-396.

Yang, K.Y., Lin, L.C., Tseng, T.Y., Wang, S.C., Tsai, T.H., 2007, Oral bioavailability of curcumin in rat and the herbal analysis from Curcuma longa by LC-MS/MS, Journal of Chromatography. $B$. Analytical Technologies in The Biomedical Life Sciences, 853(1-2): 183-189. 